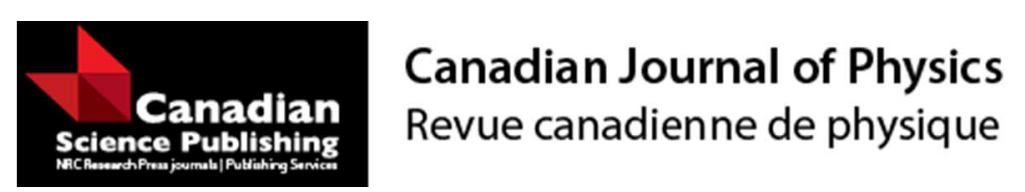

\title{
The Coefficient of Restitution of Pressurized Balls: A Mechanistic Model
}

\begin{tabular}{|r|l|}
\hline Journal: & Canadian Journal of Physics \\
\hline Manuscript ID & cjp-2015-0378.R1 \\
\hline Manuscript Type: & Article \\
\hline Date Submitted by the Author: & 31 -Aug-2015 \\
\hline Complete List of Authors: & $\begin{array}{l}\text { Georgallas, Alex; Dalhousie University, Faculty of Agriculture, Department } \\
\text { of Engineering } \\
\text { Landry, Gaëtan; Dalhousie University, Faculty of Agriculture, Department } \\
\text { of Engineering }\end{array}$ \\
\hline Keyword: & Coefficient of Restitution, Dynamics, Inflated Balls, Pressure, Impact Model \\
\hline &
\end{tabular}




\title{
The Coefficient of Restitution of Pressurized Balls:
}

\author{
A Mechanistic Model
}

\author{
Alex Georgallas and Gaëtan Landry \\ Department of Engineering, Faculty of Agriculture, \\ Dalhousie University \\ Truro, NS, B2N 5E3
}

Corresponding Author: Alex Georgallas

alex.georgallas@dal.ca

Office phone: 9028936691

Office fax: 9028931406

PACS Index Classification:

45.50.-j Dynamics and kinematics of a particle and a system of particles

Keywords: Coefficient of Restitution, Dynamics, Inflated Balls, Pressure, Impact Model. 


\section{Abstract}

Pressurized, inflated balls used in professional sports are regulated so that their behaviour upon impact can be anticipated and allow the game to have its distinctive character. However, the dynamics governing the impacts of such balls even on stationary hard surfaces can be extremely complex. In this paper examine the energy transformations which arise from the compression of the gas within the ball and from the shear forces associated with the deformation of the wall. We develop a simple mechanistic model of the dependence of the coefficient of restitution, $e$, upon both the gauge pressure, $P_{G}$, of the gas and the shear modulus, $G$, of the wall. The model is validated using the results from a simple series of experiments using three different sports balls. The fits to the data are extremely good for $P_{G}>25 \mathrm{kPa}$ and consistent values are obtained for the value of $G$ for the wall material. As far as the authors can tell, this simple, mechanistic model of the pressure dependence of the coefficient of restitution is the first in the literature. 


\section{Introduction}

The dynamical behaviour associated with bouncing pressurized (inflated) balls can be extremely complex. Even so some aspects can be readily addressed experimentally. Indeed, the question of how energy loss at impact varies with the ball's internal pressure is a popular science fair project. In most cases the data are made to fit either an exponential [1] or a power law [2] without invoking any theoretical reasons for the choice. So it came as a surprise to the authors that there appears to be no simple, mechanistic model of the pressure dependence of the coefficient of restitution in the literature. This paper sets out to remedy this void.

There is of course a considerable body of work on impact modelling methodology in general. In the more specific case of energy loss in sports ball impacts the literature is more focussed sportsrelated aspects, such as duration of impact, force exerted and 'footprint' of the collision [2-16]. For most balls used in professional sports, the internal pressure of the ball is determine by the rules of the governing body and is not a variable. Some sports bodies, such as basketball's FIBA [17], regulate the height of the bounce (and thus indirectly the pressure). Others regulate the pressure directly [18-20]. We note here that this paper does not address inflated sports balls which are non-spherical, as in the case of rugby and American football, even a recent controversy has been a popular news item of late [21]!

Impact behaviour is often characterized in terms of the coefficient of restitution $e$ which is a measure of the energy dissipated at the collision. In the theory section which follows, we develop a simple mechanistic model of the dependence of $e$ on both the internal pressure of the ball and on the forces associated with the deformation of the wall of the ball.

The experimental validation of the model is described in the subsequent section. Three different balls, a basketball, volleyball and soccer ball are dropped onto a hard floor and the coefficient $e$ is determined over a range of pressure. It is seen that the fit to the experimental data is very good, particularly for high gauge pressures. Our discussion of the shortcomings of the model and our conclusions are given in the final section. 


\section{Theory and Model}

In the case of an ideal (perfectly elastic) ball impacting a hard, stationary surface, its velocity after impact, $v_{f}$, would have exactly the same magnitude as its velocity before impact, $v_{i}$, since its kinetic energy would be unchanged. For inelastic impacts where energy losses do occur it is convenient to characterize the impact in terms of the coefficient of restitution, which may be defined through

$e=\frac{\left|v_{f}\right|}{\left|v_{i}\right|}$

Clearly $e$, which must lie between 0 and 1 , is specific to the nature of the impact and in particular to the physical characteristics of the ball. In the case of inflated sports balls both the degree of inflation and the nature of the wall material play significant roles. In this section we will develop a model that shows explicitly the dependence of $e$ on both the gauge pressure of the gas and the shear modulus of the wall material.

We view the ball as a hollow sphere of radius $R$, wall thickness $D_{W}$ and internal pressure $P_{i}$. Upon impact with a hard surface, the ball will be compressed by an amount $x$ resulting in a circle of area $A(x)$ in contact with the surface, as shown in Fig.1. The following geometrical relations hold true:

$$
\begin{aligned}
& r(x)=\left[R^{2}-(R-x)^{2}\right]^{1 / 2} \\
& A(x)=\pi\left[R^{2}-(R-x)^{2}\right]=\pi x(2 R-x) \\
& V(x)=\frac{4}{3} \pi R^{3}-\frac{1}{3} \pi x^{2}(3 R-x) \\
& \cos \theta(x)=\frac{R-x}{R}
\end{aligned}
$$

The consequences of this compression are threefold:

1. The pressure difference across the area $A(x)$ will result in a restoring force $F_{P}$.

2. Deformation along the perimeter of $A(x)$ will result in a shear strain within the wall giving rise to a restoring force $F_{W}$.

3. Dissipative forces within the wall will act during both the compression and decompression stages of the impact.

We note here that Hubbard and Stronge [11] conducted an analysis which took into account gas pressure and wall tension. However they did not address the problem of energy loss dependence 
on gauge pressure.

\subsection{The restoring force $F_{P}$ arising from the gauge pressure of the gas}

Initially the gauge pressure is $P_{G}=P_{i}-P_{0}$, where $P_{0}$ is the pressure outside of the ball. As the ball compresses the internal pressure will increase from its initial value $P_{i}$ to a value $P(x)$, with the resulting upward restoring force

$F_{P}=\left(P(x)-P_{0}\right) A(x)=\left(P(x)-P_{0}\right) \pi x(2 R-x)$

Now for an isothermal compression of the gas inside the ball:

$P(x) V(x)=P_{i} V_{i} \quad \Rightarrow \quad P(x)=\frac{P_{i} V_{i}}{V(x)}=\frac{4 R^{3}}{4 R^{3}-x^{2}(3 R-x)} P_{i}$

Resulting in

$F_{P}=\left[\frac{4 R^{3}}{4 R^{3}-x^{2}(3 R-x)} P_{i}-P_{0}\right] \pi x(2 R-x)=2 \pi R^{2} P_{G}\left[\left(\frac{x}{R}\right)-\frac{1}{2}\left(\frac{x}{R}\right)^{2}+\frac{3}{4}\left(1+\frac{P_{0}}{P_{G}}\right)\left(\frac{x}{R}\right)^{3}+\cdots\right]$

Without the higher order terms, $F_{P}$ would have the characteristics of a Hooke's Law restoring force:

$F_{P}=2 \pi R P_{G} x$

This would clearly be true in the case of small compressions $x \ll R$. However, we must be also mindful of the case where $P_{G} \rightarrow 0$. When $P_{G}=0, F_{P}=\frac{3 \pi P_{0}}{2 R} x^{3}+\cdots$, thus in dropping the higher order terms we can no longer extend the model to the case where the gauge pressure is zero. If fact equation (3) holds only for $x \ll R$ and $P_{G} \gg 0$. This approximation is further examined in the discussion in section 4.

\subsection{The restoring force $F_{W}$ arising from the deformation of the wall}

All along the perimeter of the area $A(x)$ the wall is subject to a shear stress equal to the force per unit area. In this case the area is $A_{p}$, the wall area associated with the perimeter, and is parallel to the restoring force $F_{W}$. Thus:

$\tau=\frac{F_{W}}{A_{p}}=\frac{F_{W}}{2 \pi r D_{W}}=\frac{F_{W}}{2 \pi\left[R^{2}-(R-x)^{2}\right]^{1 / 2} D_{W}}$

The shear stress may be written in terms of the shear modulus $G$ and the shear strain $\gamma$, which in this case is equal to the angle of deformation $\theta$ of the wall.

$\tau=\gamma G=\theta G=G \cos ^{-1}\left(\frac{R-x}{R}\right)$ 
So

$F_{W}=2 \pi G D_{W}\left[R^{2}-(R-x)^{2}\right]^{1 / 2} \cos ^{-1}\left(\frac{R-x}{R}\right)=4 \pi G D_{W} R\left[\left(\frac{x}{R}\right)-\frac{1}{6}\left(\frac{x}{R}\right)^{2}-\frac{1}{30}\left(\frac{x}{R}\right)^{3}+\cdots\right]$

Again this leads to a Hooke's Law force in the case of small compressions, $x \ll R$ :

$F_{W}=4 \pi G D_{W} x$

\subsection{Energy considerations at the impact}

Fig. 2 shows a schematic of the impact. For convenience we divide it into three stages: A the instant before impact, $\mathrm{B}$ the maximum compression and $\mathrm{C}$ the instant after impact. During this impact the net restoring force $F_{R}$ acting on the ball is

$F_{R}=\left(2 \pi R P_{G}+4 \pi G D_{W}\right) x=k x$

This Hooke's Law force has an associated potential energy $1 / 2 k x^{2}$.

Let us suppose that the ball is subject to the same average dissipative force $F_{D}$ upon both compression and decompression. The nature of the mechanisms of energy dissipation is addressed in the discussion in section 4 . The energy transformations during compression $\mathrm{A} \rightarrow \mathrm{B}$ may be written:

$K_{i}=1 / 2 k x^{2}-m g x+F_{D} x$

Where $K_{i}$ is the initial kinetic energy of the ball, $m$ is the mass of the ball and $g$ the acceleration due to gravity. Likewise during the decompression, $\mathrm{B} \rightarrow \mathrm{C}$

$K_{f}=e^{2} K_{i}=1 / 2 k x^{2}-m g x-F_{D} x$

where $K_{f}$ is the final kinetic energy of the ball. The sum and difference of these give

$K_{i}\left(1+e^{2}\right)=k x^{2}-2 m g x$

$K_{i}\left(1-e^{2}\right)=2 F_{D} x$

Leading to the ratio

$\frac{\left(1+e^{2}\right)}{\left(1-e^{2}\right)^{2}}=\frac{\left(k x^{2}-2 m g x\right) K_{i}}{4 F_{D}^{2} x^{2}} \approx \frac{k K_{i}}{4 F_{D}^{2}}$

This approximation, $1 / 2 k x^{2} \gg m g x$, holds true in most situations since the energy stored in the compressed ball is very much greater than the small change in its gravitational potential. It is certainly true for any ball stiff enough to support its own weight at zero gauge pressure without being significantly deformed. Using (5) we can write the final form of the model. 
$\frac{\left(1+e^{2}\right)}{\left(1-e^{2}\right)^{2}}=\frac{2 \pi R K_{i} P_{G}+4 \pi G D_{W} K_{i}}{4 F_{D}^{2}}$ or $\frac{\left(1+e^{2}\right)}{\left(1-e^{2}\right)^{2}}=A P_{G}+B$

with

$A=\frac{\pi R K_{i}}{2 F_{D}^{2}} ; \quad B=\frac{\pi G D_{W} K_{i}}{F_{D}^{2}} ; \quad \frac{B}{A}=\frac{2 G D_{W}}{R}$

In the experimental verification of this model, $e$ is determined for successive values of $P_{G}$. The quantities $A$ and $B$ can be treated as adjustable parameters which may be obtained by fitting to equation (6). This is done in the following section.

\section{Experimental Validation}

For validation of the model, three different types of inflated sports balls were utilized, a basketball, a soccer ball and volleyball, the details of which are given in Table 1. The balls were systematically bounced off a hard floor under laboratory conditions. Two dropping heights $(1.5 \mathrm{~m}$ and $0.75 \mathrm{~m})$ were chosen for a range of gauge pressure from 0 to $100 \mathrm{kPa}$.

There are several methods in common use to measure the coefficient of restitution [22-25]. We chose to release the ball from a known height $H$ and then use a microphone to determine the time of flight $t_{f}$ from the first bounce to the second. If we assume that air resistance is negligible, $e$ is determined from

$e=\frac{\left|v_{f}\right|}{\left|v_{i}\right|}=\frac{1 / 2 g t_{f}}{\sqrt{2 g H}}=\sqrt{\frac{g t_{f}^{2}}{8 H}}$

Pressure was changed by inflating the ball manually with a pump and measured with a pressure sensor. The basketball and volleyball were each released 5 times, and the pressure was measured before and after each trial to ensure there were no for pressure changes during the course of a trial. The soccer ball was released 9 times for the 4 lowest pressure trials, to accommodate the polyhedral nature of the ball at these low pressures. At higher pressures the ball was essentially spherical. The fits to equation (6) for each ball are presented in Fig.3, whilst the ratio of the measured to the predicted value of $e$ is are shown in Fig.4. The values of the fitted parameters of equation (7) are tabulated in Table 2. 


\section{Discussion}

Two observations are immediately apparent upon review of the results. The first is that the model fits the data extremely well for $P_{G}>25 \mathrm{kPa}$. The second is that very consistent values are obtained for the shear modulus $G$ of the wall material of the balls. Although direct comparisons are difficult owing to the composite nature of the material, it is worthwhile comparing to some common elastic materials such as rubber which has $G$ ranging from $3 \times 10^{5}$ to $17 \times 10^{5} \mathrm{~Pa}[26]$. Understandable and consistent values are also obtained for the dissipative force $F_{D}$. Table 2 shows that the dissipative force is greater when the ball is dropped from the greater height as expected (since the area of deformation of the ball is greater). The energy dissipation takes place principally in the form of heat during the bending and unbending of the wall and subsequent wall vibrations [3]. Clearly at higher gauge pressures both the magnitude and duration of the compression of the ball is less, resulting in less dissipation.

The coefficient of restitution would be affected by other external variables such as altitude and temperature. In our model the coefficient is expressed directly in terms of the gauge pressure. Altitude would be relatively easy to incorporate using the barometric pressure equation. However temperature would be much more difficult, since it would affect the thermal properties of the ball material including thermal conductivity, expansion and shear modulus (stiffness) [16]. Why the fit is not so good at low pressure $\left(P_{G}<25 \mathrm{kPa}\right)$ has in part already been addressed in the derivation of equation (3). At low pressure the ball will more easily deform, and the assumption that $x \ll R$ is no longer true. Indeed at these low pressures a deformation of about $x=2 \mathrm{~cm}$ was readily observable, giving rise to $x / R=0.2$ for the balls used in this validation. In examining the expansion leading to equation (3) we see that for this value of $x / R$ the third order term (containing $\left.P_{0} / P_{G}\right)$ gives $\frac{3}{4}\left(1+\frac{P_{0}}{P_{G}}\right)(0.2)^{2}=0.15$ for $P_{G}=25 \mathrm{kPa}$ (where we have taken atmospheric pressure $P_{0}=101.325 \mathrm{kPa}$ ). Thus ignoring this term becomes progressively less tenable as $P_{G}$ decreases. This can be seen in fig 4 where above $P_{G}=25 \mathrm{kPa}$ the spread between observed and predicted values of $e$ is less than $2.5 \%$.

Clearly better agreement would be obtained if the restoring forces (3) and (4) were not truncated, which would the lead to a more complex relationship between $e$ and $x$. However this was not the intent of this model, which was to derive a closed form expression for $e$ as a function of the gauge pressure $P_{G}$ in the range normally associated with inflated sports balls. 


\section{Acknowledgements}

One of us (GL) would like to thank Simon de Vet of Dalhousie University for fruitful discussions concerning time of flight methods.

\section{References}

1 K. Osman and B. S. Kim. ISB J. Phys. 3(2), 2 (2009).

2 N. J. Bridge. Phys. Educ. 33(3), 174 (1998). doi:10.1088/0031-9120/33/3/018.

3 A. D. C. Ashcroft and W. J. Stronge. "Dynamic dissipation during bounce of tennis ball". Proceedings of the 5th International Conference on the Engineering of Sports (Davis, California). Volume 1, p. 386 (2004).

4 M. J. Carré, D. M. James, S. J. Haake. Proc. Inst. Mech. Eng. C.: J. Mech. Eng. Sci. 218(3), 273 (2004). doi:10.1243/095440604322900408.

5 R. Cross. Am. J. Phys. 67(3), 222 (1999). doi:10.1119/1.19229.

6 R. Cross. Am. J. Phys 68(11), 1025 (2000). doi:10.1119/1.1285945.

7 R. Cross. Sports Eng. 17(4), 239 (2014). doi:10.1007/s12283-014-0159-x.

$8 \quad$ R. Cross. Am. J. Phys. 82, 189 (2014). doi:10.1119/1.4839055.

9 S. P. Hendee, R. M. Greenwald, and J. J. Crisco. J. Appl. Biomech. 14(4), 390 (1998).

10 Y. Honda. "Normal impact of hollow balls on flat surfaces" The Engineering of Sport 6, Volume 1, p. 367 (2006). doi:10.1007/978-0-387-46050-5 65.

11 M. Hubbard and W. J. Stronge. Sports Eng. 4, 49 (2001) doi:10.1046/j.14602687.2001.00073.x.

12 D. M. James, M. J. Carré, and S. J. Haake. "The normal impact of a cricket ball on a cricket pitch". Proceedings of the 5th International Conference on the Engineering of Sports (Davis, California). Volume 2, p. 66. (2004).

13 J. Njock-Libii (2011). "AC 2011-169: Applying Dynamics to the Engineering of the Perfect Bounce: Experimental Investigation of Why the NBA Requires a Specific Inflation Pressure for Basketballs Used in Professional Games". Proceedings of the 2011 ASEE Annual Conference, (Vancouver). Available at 
https://www.asee.org/public/conferences/1/papers/169/view, accessed 2015-06-02.

14 J. Njock-Libii (2012). "AC 2012-2947: Applying Dynamics to the Bouncing of Game Balls: Experimental Investigation of the Relationship Between the Duration of a Linear Impulse During an Impact and the Energy Dissipated". Proceedings of the 2012 ASEE Annual Conference (San Antonio). Available at https://www.asee.org/public/conferences/8/papers/2947/view, accessed 2015-06-02.

15 W. J. Stronge and A. D. C. Ashcroft. Int. J. Impact Eng. 34, 1003 (2007). doi:10.1016/j.ijimpeng.2006.04.006.

16 N. Wiart, J. Kelley, D. James, and T. Allen. Proc. Inst. Mech. Eng. P.: J. Sport Eng. Tech. 225(4), 189 (2011), doi:10.1177/1754337111411644.

17 Official Basketball Rules 2014 - Basketball Equipment. Fédération Internationale de Basketball. 2014. pp.11-12. Available at http://www.fiba.com/downloads/Rules/2014/BasketballEquipment2014_Final_V1_withC overs_LOW.pdf, accessed 2015-06-02.

18 Official Rules of the National Basketball Association: 2014-2015. National Basketball Association. 2014. p.10. Available at https:/turnernbahangtime.files.wordpress.com/2014/12/2014-15-nba-rule-book.pdf, accessed 2015-06-02.

19 Laws of the Game: 2014-2015. Fédération Internationale de Football Association. 2014. p.15. Available at http://www.fifa.com/mm/Document/FootballDevelopment/Refereeing/02/36/01/11/27 06 2014 new--LawsofthegamewebEN_Neutral.pdf, accessed 2015-06-02.

20 Official Volleyball Rules: 2015-2016. Fédération International de Volleyball. 2014. p.16. Available at http://www.fivb.org/EN/Refereeing-

Rules/documents/FIVB Volleyball Rules 2015-2016 EN V3 20150205.pdf, accessed 2015-06-02.

21 G. Botelho and M Castillo. 'Deflategate:' 4-game suspension for Tom Brady, \$1M fine for Pats. CNN. 2015. Available at http://edition.cnn.com/2015/05/11/us/nfl-deflategateresponse/, accessed 2016-06-08.

22 A. Haron and K. A. Ismail. IOP Conf. Ser.: Mater. Sci. Eng. 36, 012038 (2011). doi:10.1088/1757-899X/36/1/012038. 
23 A. D. Bernstein. Am. J. Phys 45(1), 41 (1977). doi:10.1119/1.10904.

24 I. Stensgaard and E. Lægsgaard. Am. J. Phys. 69(3), 301 (2001). doi:10.1119/1.1326077.

25 C. E. Aguiar and F. Laudares. Am. J. Phys. 71(5), 499 (2001). doi:10.1119/1.1524166.

26 J. M. Hill and A. I Lee. Q. J. Mech. Appl. Math. 42(2), 267 (1989). doi:10.1093/qjmam/42.2.267. 


\begin{tabular}{|c|c|c|c|c|c|}
\hline \multirow{2}{*}{ Ball } & Manufacturer & Model & $\begin{array}{r}\text { Mass } m \mathrm{~kg} \\
\left(\text { at } P_{\mathrm{g}}=0\right) \\
\pm 0.0001\end{array}$ & $\begin{array}{c}\text { Radius } R \mathrm{~m} \\
\left(\text { at } P_{\mathrm{g}}=0\right) \\
\pm 0.0015\end{array}$ & $\begin{array}{c}\text { Wall } \\
\text { thickness } \\
D_{w} \mathrm{~m}\end{array}$ \\
\hline Basketball & Wilson & WTB0935 & 0.5929 & 0.1175 & $3.10 \pm 0.09$ \\
\hline Soccer ball & Nike & SC2400-471 & 0.4222 & 0.1080 & $4.51 \pm 0.08$ \\
\hline Volleyball & Wilson & WTH3501 & 0.2712 & 0.1035 & $5.07 \pm 0.28$ \\
\hline
\end{tabular}

Table 1

\section{CAPTION FOR TABLE 1}

Details of the sports balls used in this study. 


\begin{tabular}{|c|c|c|c|c|c|}
\hline \multirow{2}{*}{ Ball } & $\begin{array}{c}\text { Height } H \\
\mathrm{~m}\end{array}$ & $\begin{array}{c}A \\
10^{4} \mathrm{~Pa}^{-1}\end{array}$ & $B$ & $\begin{array}{c}G \\
10^{5} \mathrm{~Pa}\end{array}$ & $\begin{array}{c}F_{D} \\
\mathrm{~N}\end{array}$ \\
\hline \multirow{3}{*}{ Basketball } & 0.75 & $2.576 \pm 0.052$ & $1.35 \pm 0.28$ & $1.01 \pm 0.27$ & $55.92 \pm 0.93$ \\
\cline { 2 - 6 } & 1.50 & $1.916 \pm 0.036$ & $1.29 \pm 0.20$ & $1.29 \pm 0.27$ & $91.7 \pm 1.5$ \\
\hline \multirow{3}{*}{ Soccer ball } & 0.75 & $2.76 \pm 0.11$ & $6.60 \pm 0.65$ & $2.90 \pm 0.49$ & $43.8 \pm 1.2$ \\
\cline { 2 - 7 } & 1.50 & $2.277 \pm 0.088$ & $4.74 \pm 0.50$ & $2.52 \pm 0.44$ & $68.1 \pm 1.8$ \\
\hline \multirow{3}{*}{ Volleyball } & 0.75 & $3.14 \pm 0.14$ & $3.50 \pm 0.80$ & $1.18 \pm 0.39$ & $32.16 \pm 0.93$ \\
\cline { 2 - 6 } & 1.50 & $2.091 \pm 0.074$ & $3.24 \pm 0.46$ & $1.62 \pm 0.39$ & $55.7 \pm 1.4$ \\
\hline
\end{tabular}

Table 2

\section{CAPTION FOR TABLE 2}

The values of the fitted parameters $A$ and $B$. The value of the shear modulus $G$ for the wall material is found from the ratio $B / A$, as shown in equation (7). 


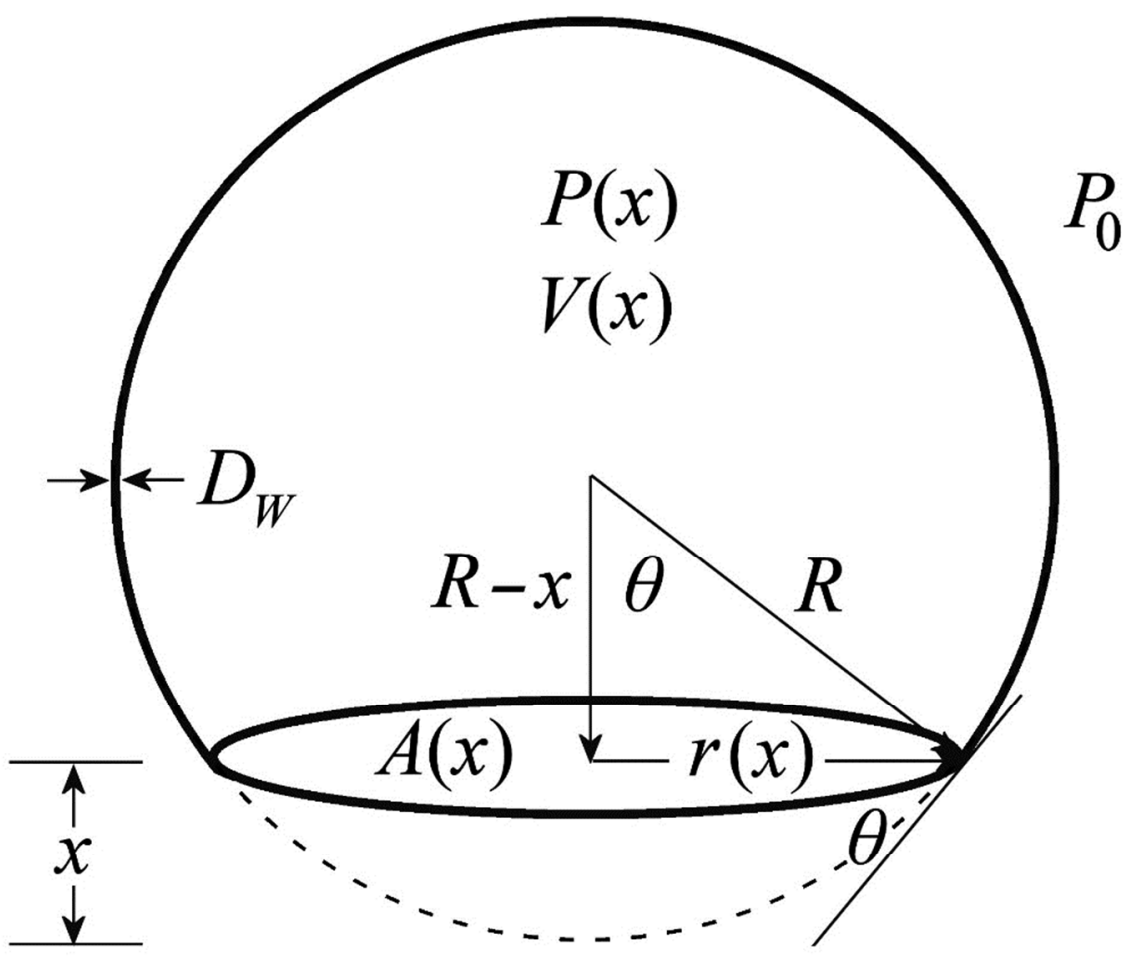

Fig.1

CAPTION FOR FIG.1:

The geometry of the compressed ball. 


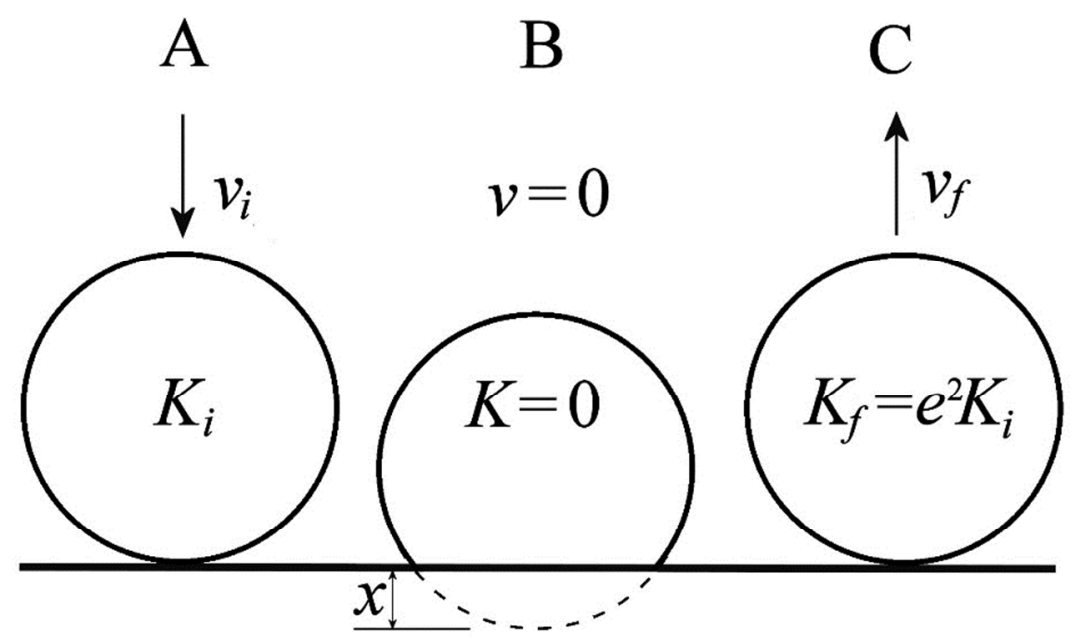

Fig.2

CAPTION FOR FIG.2:

A schematic of the impact of the ball on a hard, stationary surface. 


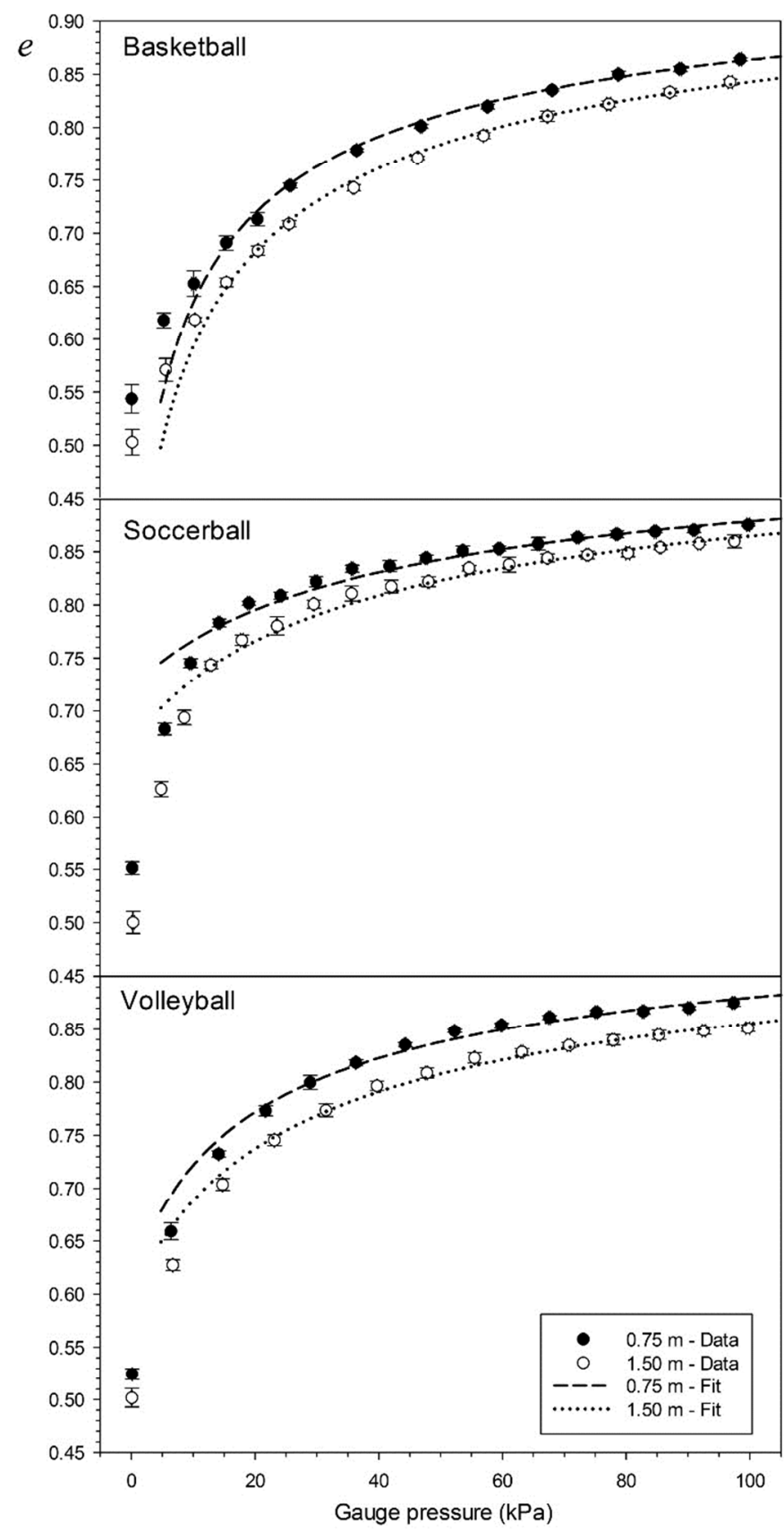

Fig.3

\section{CAPTION FOR FIG.3:}

The experimental data for each ball for each of the two dropping heights. The error bars represent the standard deviation for the trials. The fits to equation (6) are shown by the broken lines. Note that the points for which $P_{G}=0$ were not included in the fits. 


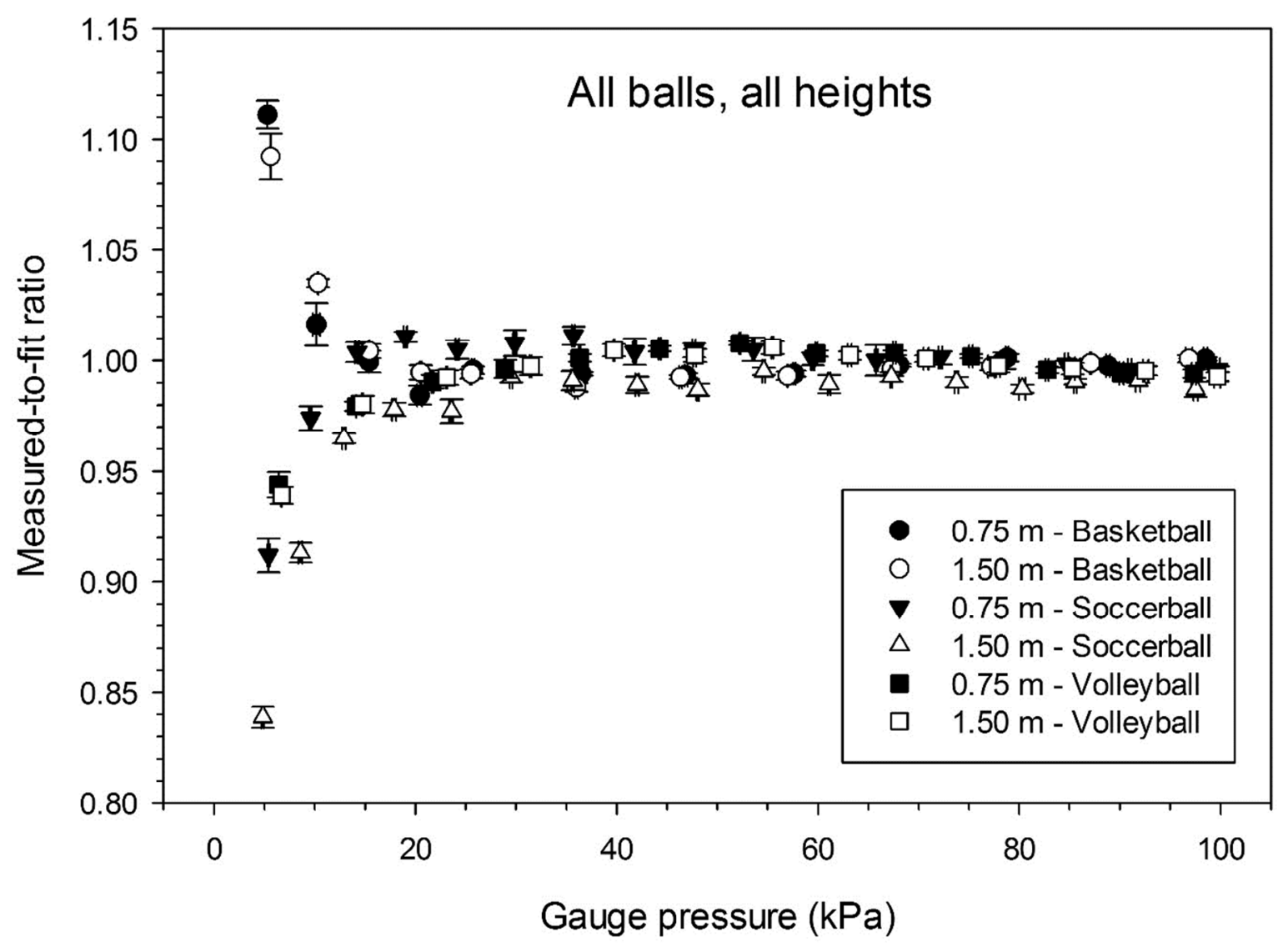

Fig.4

\section{CAPTION FOR FIG.4:}

The ratio of the predicted to the measured value of $e$ for all balls and all heights as a function of gauge pressure. The fits are particularly good for $P_{G}>25 \mathrm{kPa}$, where the spread between observed and predicted values of $e$ is less than $2.5 \%$, with no individual point lying further than $1.5 \%$ from the predicted value. 